\title{
Imaging of sensorineural hearing loss: a pattern-based approach to diseases of the inner ear and cerebellopontine angle
}

\author{
Berit M. Verbist
}

Received: 31 May 2011 /Revised: 27 September 2011 /Accepted: 11 October 2011 /Published online: 9 December 2011

(C) The Author(s) 2011. This article is published with open access at Springerlink.com

\begin{abstract}
An overview is presented of the common and uncommon diseases of the inner ear and of the cochleovestibular nerve within the internal acoustic meatus and cerebellopontine angle cistern causing sensorineural deafness.

An imaging-pattern-based approach is used to help detect disease and narrow the differential diagnosis.

Main Messages

- The most common soft tissue mass lesions in the cerebellopontine angle are schwannoma and meningioma.

- Contrast-enhanced MRI may reveal clinically unsuspected inflammatory, auto-immune or tumoural disease.

- Hearing loss may be caused by infection, inflammation or, rarely, perineural tumour spread along the cochleovestibular nerve.

- Labyrinthitis may lead to rapidly progressive ossification of the labyrinth.
\end{abstract}

Keywords Imaging · Computed tomography · Magnetic resonance imaging $\cdot$ Inner ear Cerebellopontine angle . Hearing loss $\cdot$ Pictorial review

\footnotetext{
B. M. Verbist $(\square)$

Department of Radiology, Leiden University Medical Center, PO Box 9600, 2300 RC,

Leiden, The Netherlands

e-mail: b.m.verbist@lumc.nl

\section{B. M. Verbist}

Department of Radiology, Radboud University

Nijmegen Medical Centre,

PO Box 9101, $6500 \mathrm{HB}$,

Nijmegen, The Netherlands
}

\section{Introduction}

Sensorineural hearing loss points at a malfunction of the inner ear or a retrocochlear condition that affects the cochleovestibular nerve within the internal acoustic meatus and cerebellopontine angle or that involves the central auditory pathway. Imaging is performed to detect or rule out congenital, infectious, inflammatory or tumoural pathology. Determining the etiology of hearing loss is helpful to establish treatment regimens that may prevent or slow down loss of auditory function. It may also contribute to counselling of the patient and - in genetic causes - his or her family.

When reading imaging studies of these patients, an analytical pattern-based approach will help to prevent uncommon or subtle disease to be overlooked and to narrow the differential diagnosis, if applied in conjunction with medical history and clinical findings.

\section{Inner ear}

Imaging of the inner ear is performed with computed tomography (CT) and/or magnetic resonance imaging (MRI). These techniques render complementary information. In patients requiring anaesthesia, these exams should be planned in one session. CT forms an ideal means to evaluate the bony details of the otic capsule and labyrinth and will also allow evaluating the course of the facial nerve canal and eventual associated anomalies of middle ear structures and the external auditory canal. MRI will render supplementary information on the fine intralabyrinthine structures.

Temporal bone CT should consist of both axial and coronal images. With multidetector CT, images can be reconstructed in any desired plane, obviating the need for direct coronal scanning. Contiguous or overlapping sections 

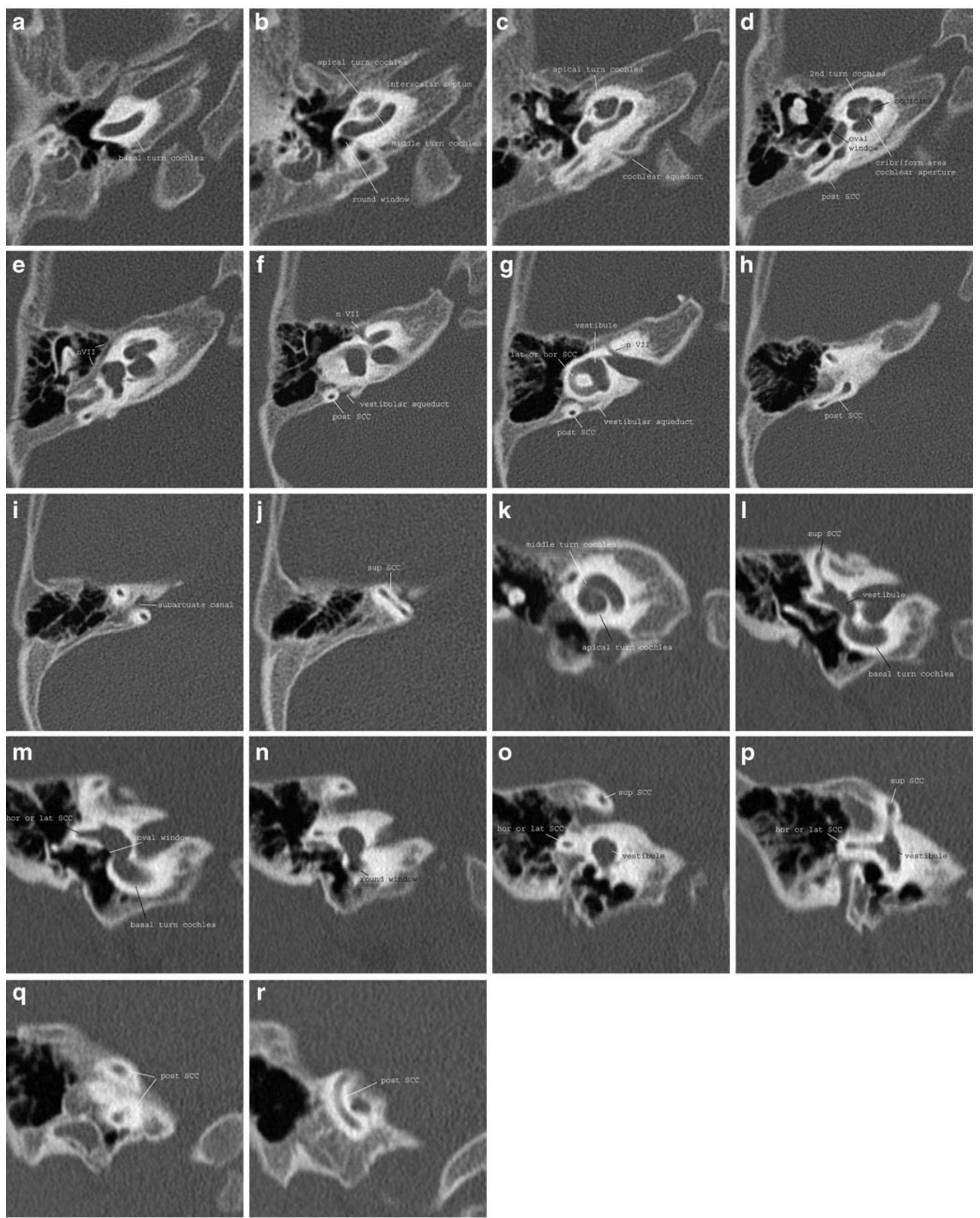

Fig. 1 Normal anatomy of the inner ear on CT. a-j Axial images; k-r coronal images 
from the superior most mastoid air cells to the stylomastoid foramen should be obtained with the gantry angle parallel to the infraorbital-meatal line. The display slice thickness should not exceed $1.5 \mathrm{~mm}$ [1].

\section{Anatomy}

The inner ear comprises the cochlea, the vestibular system and the endolymphatic duct and sac. The normal cochlea consists of two-and-a-half turns separated by interscalar septae. Within each turn the cochlea is divided into the scala tympani and scala vestibuli by the osseous spiral lamina. These scalae are connected to each other at the apical tip of the cochlea (helicotrema) and they contain perilymph. The scala media, containing the organ of Corti and filled with endolymph, cannot be visualised separately with current imaging systems. The modiolus containing the spiral ganglion can, however, be visualised in the centre of the cochlea. The cochlea is connected to the middle ear by the round window. The vestibular system is located posterior to the cochlea and contains the vestibule with the saccule and utricle and three semicircular canals which are oriented perpendicular to each other. The vestibule harbours the oval window; the stapes footplate is attached to the oval window. The endolymphatic duct is connected to the saccule and courses through a bony canal - the vestibular aqueduct - to form the endolymphatic sac in the posterior fossa (Figs. 1 and 2).

\section{Imaging patterns of disease}

\section{Abnormal development}

Formation of the labyrinth starts at about 3.5 weeks of gestation by formation of the otic disc and is complete at about 26 weeks of gestation. The otic disc becomes invaginated and gives rise to the otic vesicle or otocyst.
The otocyst divides into dorsal and ventral segments that will become vestibular and auditory parts; the endolymphatic appendage appears dorsally. The mesenchyme surrounding the otocyst becomes condensed and forms the otic capsule [2].

Abnormal development may cause a myriad of variations, ranging from complete absence of the labyrinth to a minor dysplasia of one semicircular canal (Fig. 3). Both ears may be affected to a different degree. Congenital malformations may be acquired, e.g. after a gestational viral infection such as by rubella or cytomegalovirus, due to a genetic cause or the result of a combination of both, as in aminoglucoside-induced deafness with underlying mitochondrial DNA mutations [3].

Genetic deafness may be sporadic or hereditary. The mode of inheritance may be X-linked, dominant or recessive and the mutation may be chromosomal or mitochondrial. In syndromal cases, typical features of inner ear dysplasia and/or associated anomalies may be seen on imaging (Fig. 4).

Several classification systems have been proposed to group patients with a distinct radiographic pattern [4-7]. Jackler et al. [5] hypothesised that dysplasias result from a developmental arrest during varying stages of inner ear organogenesis and identified the following groups: labyrinthine aplasia, cochlear aplasia, cochlear hypoplasia, common cavity malformation and incomplete partitioning. The latter may be divided into cystic cochleovestibular dysplasia (incomplete partitioning type I) and incomplete partitioning type II (true Mondini malformation) [6]. This is a useful framework to describe and understand labyrinthine dysplasias, however more variations exist. A report should contain a precise description of the aspect of the cochlea, modiolus, cochlear aperture, vestibule and SCCs, the vestibular aqueduct and eventual associated anomalies of the middle ear or external auditory canal in order to make a proper plan for hearing rehabilitation and patient counseling.
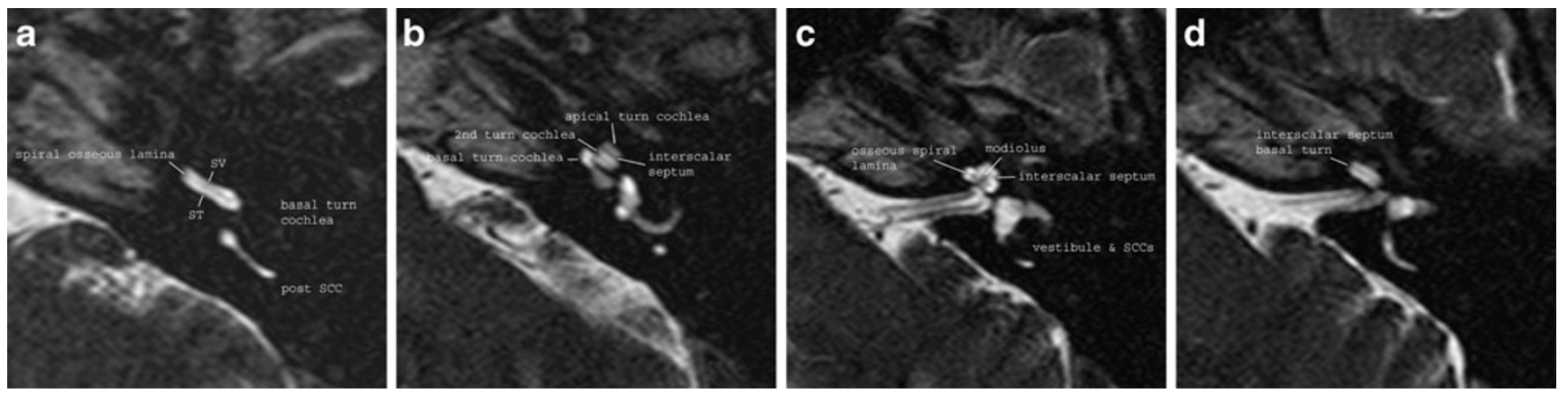

Fig. 2 Normal anatomy of the cochlea on MRI. a-d Three-dimensional T2-weighted turbo spin echo (TSE) images through the left cochlea 

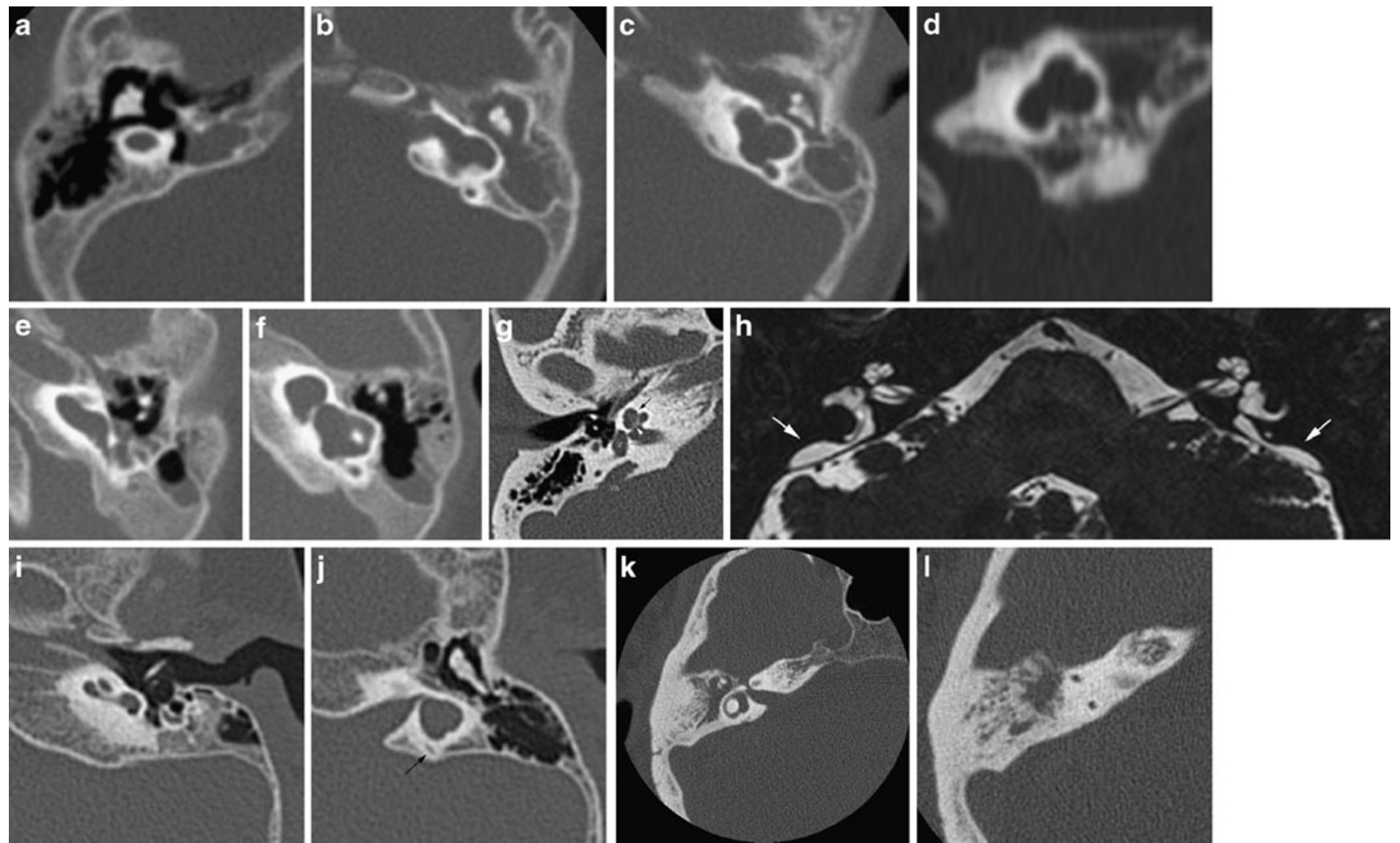

Fig. 3 Inner ear dysplasias. a-d Patient 1: a 6-month-old girl presenting with congenital deafness has bilateral congenital malformations of the labyrinth on CT. On the right (a) axial CT shows a small cyst in place of the cochleovestibular system, corresponding to labyrinthine aplasia. The internal auditory canal (IAC) is also absent. On the left, axial $(\mathbf{b}, \mathbf{c})$ and coronal (d) CT shows an IAC bending posteriorly towards a cystic dilated vestibular system. There is no development of the cochlea anteriorly; hence this developmental anomaly can be classified as a cochlear aplasia. e, f Patient 2: on axial CT images of this deaf child, a widened cochlea without a modiolus renders a cystic appearance of the cochlea. The vestibule also shows pronounced enlargement. This has been classified as a cystic cochleovestibular dysplasia (incomplete partition type I). $\mathbf{g}$, h Patient 3 shows a classic Mondini malformation (incomplete partition type II) consisting of a cochlea with a normal basal turn, but confluent middle and apical turn (black arrow), a widened vestibule (V) and an enlarged vestibular aqueduct (VA) (the normal size of the VA is $1-1.5 \mathrm{~mm}$ at its midpoint and generally does not exceed the size of the SCC). The modiolus is deficient (white arrowhead). On a 3D T2 TSE MR image the enlarged endolymphatic duct and sac are well seen (white arrows). i, j Patient 4: in this patient a foreshortened cochlea and a cystic appearance of the vestibular system is seen without enlargement of the vestibular aqueduct (black arrow). This dysplasia is more difficult to identify within the current classification system. k, I Patient 5: absence of the posterior semicircular canal illustrates that the abnormal development may be limited to the semicircular canals. Isolated absence of the posterior SCC may be seen in Waardenburg or Allagile syndrome
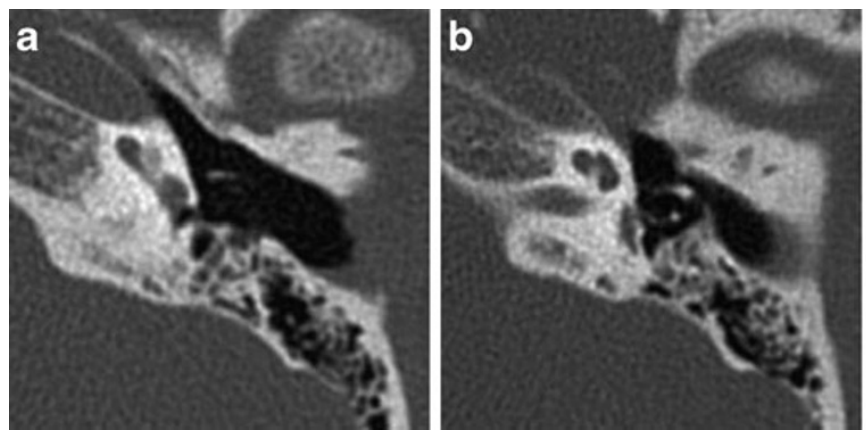

Fig. 4 Syndromal inner ear dysplasia. a-d Middle and inner ear malformations in a patient with CHARGE: axial CT images from cranial to caudal show dysplastic ossicles, a narrow oval and round window, a short cochlea with incomplete partitioning and agenesis of
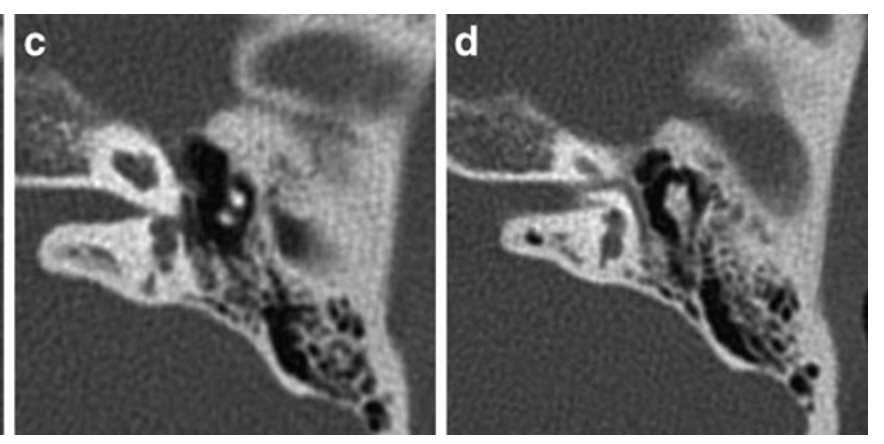

the semicircular canals. These are particular features for CHARGE. Furthermore associated ophtalmic (coloboma) and nasal (choanal atresia) abnormalities were present 

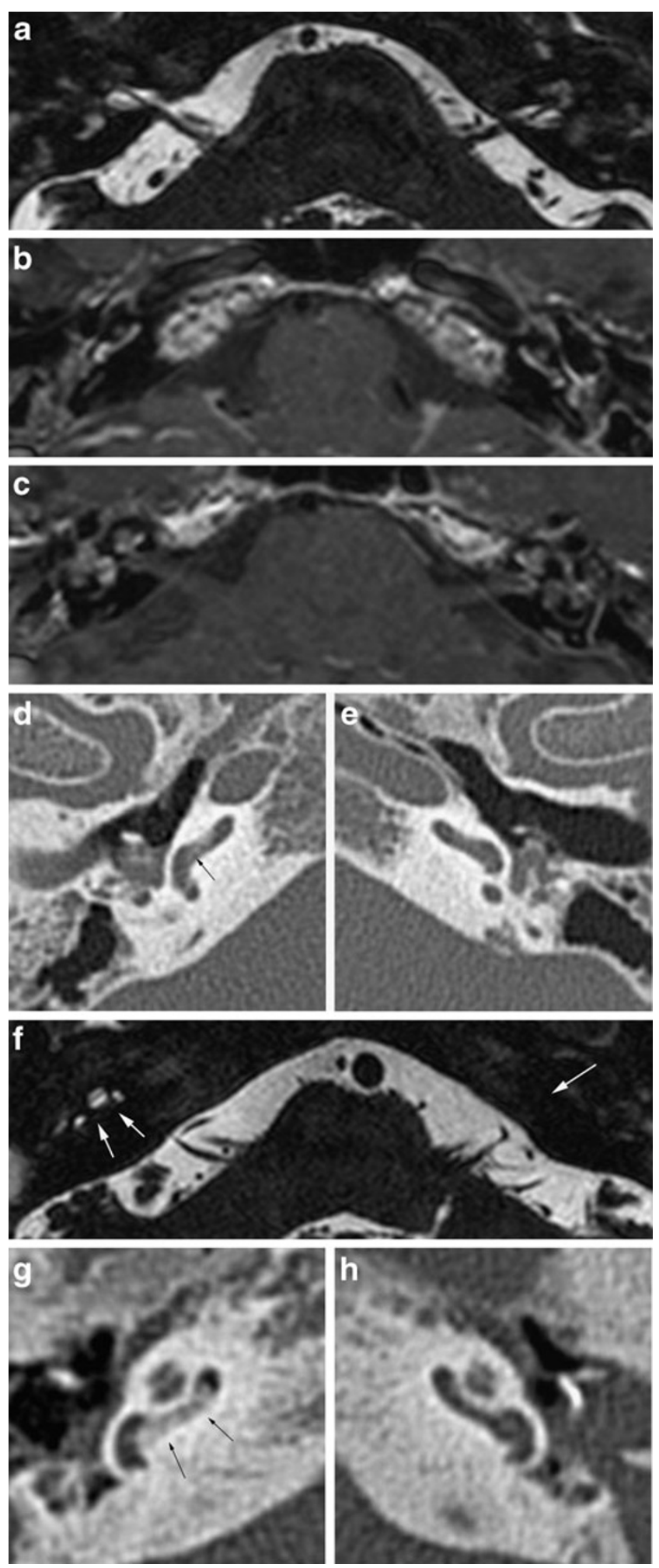

Fig. 5 Labyrinthitis. a-e Patient 1 developed acute, severe hearing loss while suffering from meningitis. On 3D T2 TSE MRI (a) bilateral signal loss of the cochleovestibular system is seen, suggestive of obliteration by inflammatory cells and fibroblasts. Contrast-enhanced T1 weighted images $(\mathbf{b}, \mathbf{c})$ show diffuse, irregular enhancement due to ongoing inflammation. Corresponding CT images show slightly increased density in the basal turn of the right cochlea (arrow in d) due to starting bone formation. The left ear (e) appears normal on CT. f-h Patient 2 had undergone several surgical procedures for chronic otitis media and cholesteatoma in the past. Three-dimensional T2 TSE image shows signal loss in the basal turn of the right cochlea and complete obliteration of the left inner ear (white arrows in f). CT confirms bony obliteration in the proximal basal turn of the right cochlea (black arrows in $\mathbf{g}$ ), but no signs of obliteration on the left (h)

\section{Abnormal density or signal intensity}

Abnormal density or signal intensity of the inner ear may be due to obliteration of the labyrinth by infectious or inflammatory changes, involvement by a bone dysplasia, a tumour, or may result from intracochlear haemorrhage. All of these will cause altered density or signal of the labyrinth and some will show enhancement. The imaging aspect and clinical history will be helpful in differentiating these conditions.

Labyrinthitis Labyrinthitis may be of infectious (bacterial, viral), inflammatory or auto-immune origin, and the spread of disease may occur either contiguously through the middle ear, cerebrospinal fluid and meninges or haematogenously. It may also be seen as an inflammatory reaction after a translabyrinthine fracture.

In labyrinthitis the perilymphatic space will become filled with inflammatory cells. At this stage changes are still

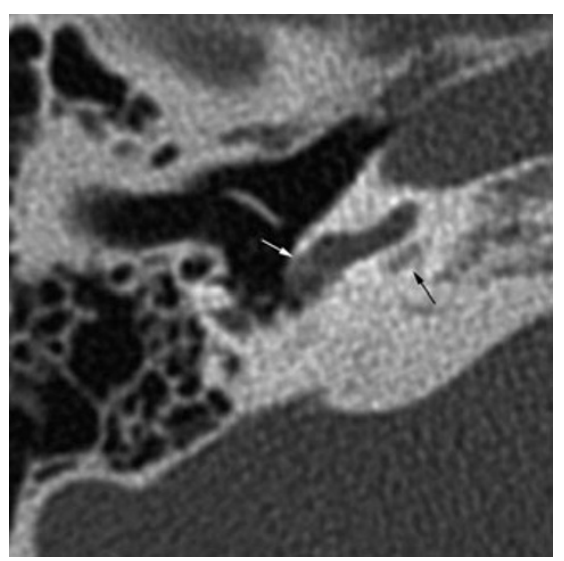

Fig. 6 Otosclerosis. Otospongiotic bone formation has caused obliteration of the round window niche (white arrow). A retrocochlear lytic focus confirms the diagnosis of fenestral and retrofenestral otosclerosis 
reversible. Eventually fibroblastic proliferation may occur, followed by bone formation, leading to permanent partial or complete obliteration of the inner ear. Rapid progression and some overlap of these stages may be seen. But, not all patients will run through all stages. The disease is considered a medical emergency at the acute stage because of the possible rapid progression to irreversible obliteration of the cochlea, which might preclude the patient from cochlear implantation, considered in case of severe or complete sensorineural hearing loss $[8,9]$.

Labyrinthine enhancement is seen in the acute stage. This may be difficult to appreciate when the labyrinth shows high signal intensity on the native T1-weighted images due to high protein content of inflammatory tissue. Once fibroblasts or osseous tissue fills the labyrinth, signal loss will be seen on thin-sliced three-dimensional (3D) T2weighted images. Enhancement may persist in the presence of fibro-osseous changes, reflecting ongoing inflammatory reaction. The enhancement is diffuse or patchy and will decrease on follow-up scans. On CT, only osseous
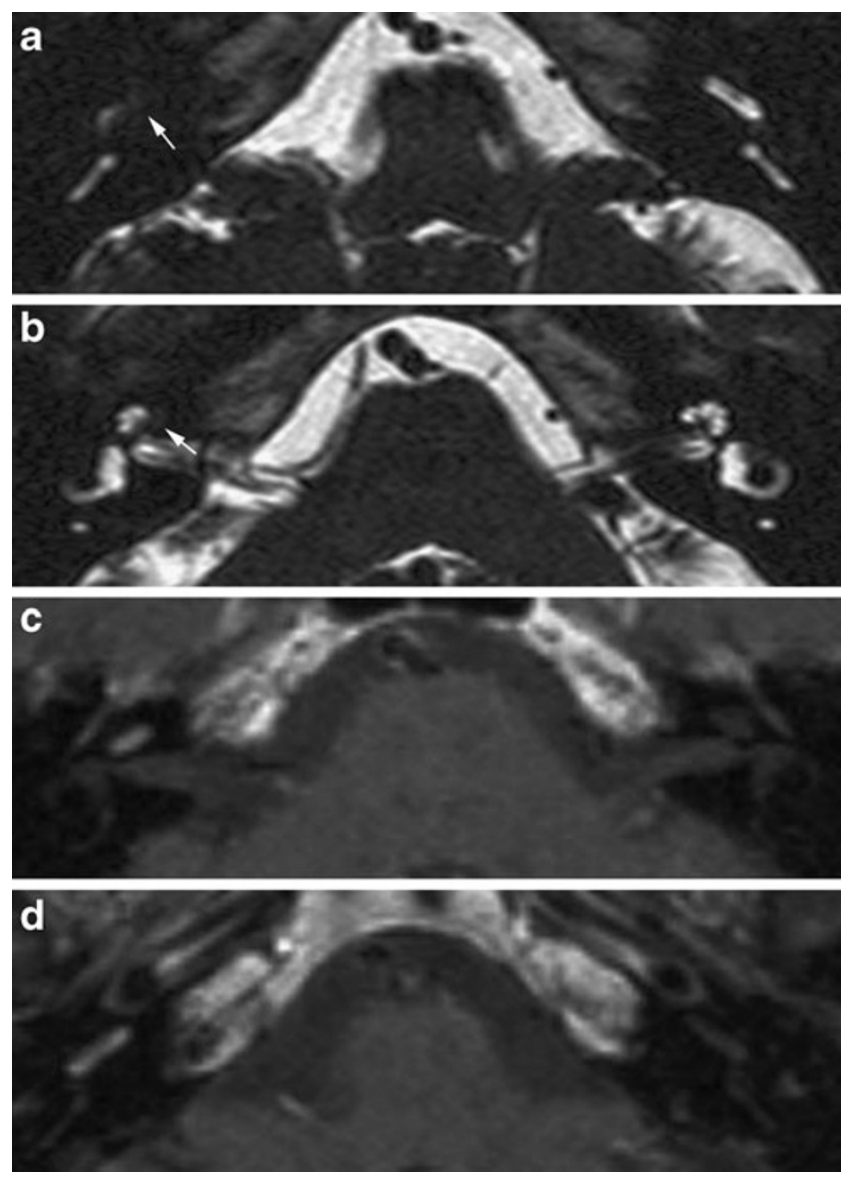

Fig. 7 Intracochlear schwannoma. A focal signal loss on 3D T2TSE images (a, b) with intense enhancement in a patient with slowly progressive hearing loss is indicative of an intracochlear schwannoma changes will be seen and the extent of obliteration may be underestimated (Fig. 5).

Otosclerosis The most common bone dysplasia affecting the temporal bone is otosclerosis. This disease is exclusive to the otic capsule and may affect the patency of the cochlea by sclerosis of the round window membrane, otospongiotic foci extending into the cochlea or secondary to complicated stapedotomy. Otosclerotic involvement of the cochlea is usually limited to the proximal part of the scala tympani [10].
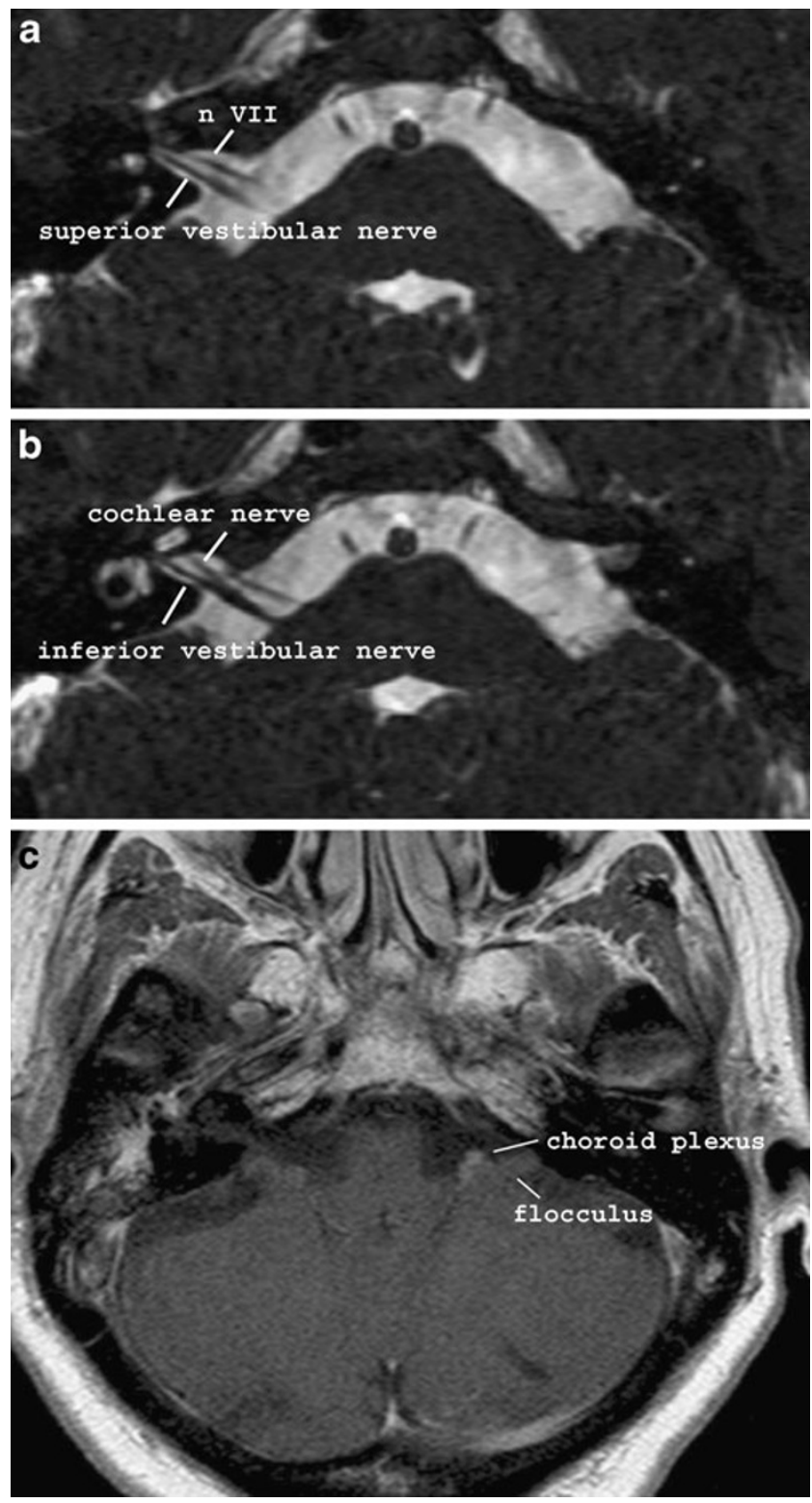

Fig. 8 Normal anatomy of the IAC and CPA on MRI. a, b The facial and cochleovestibular nerve are well seen on a thin-slice 3D T2weighted sequence. $\mathbf{c}$ Contrast-enhanced $\mathrm{T} 1$ shows the flocculus of the cerebellum and the enhancing choroid plexus within the CPA 

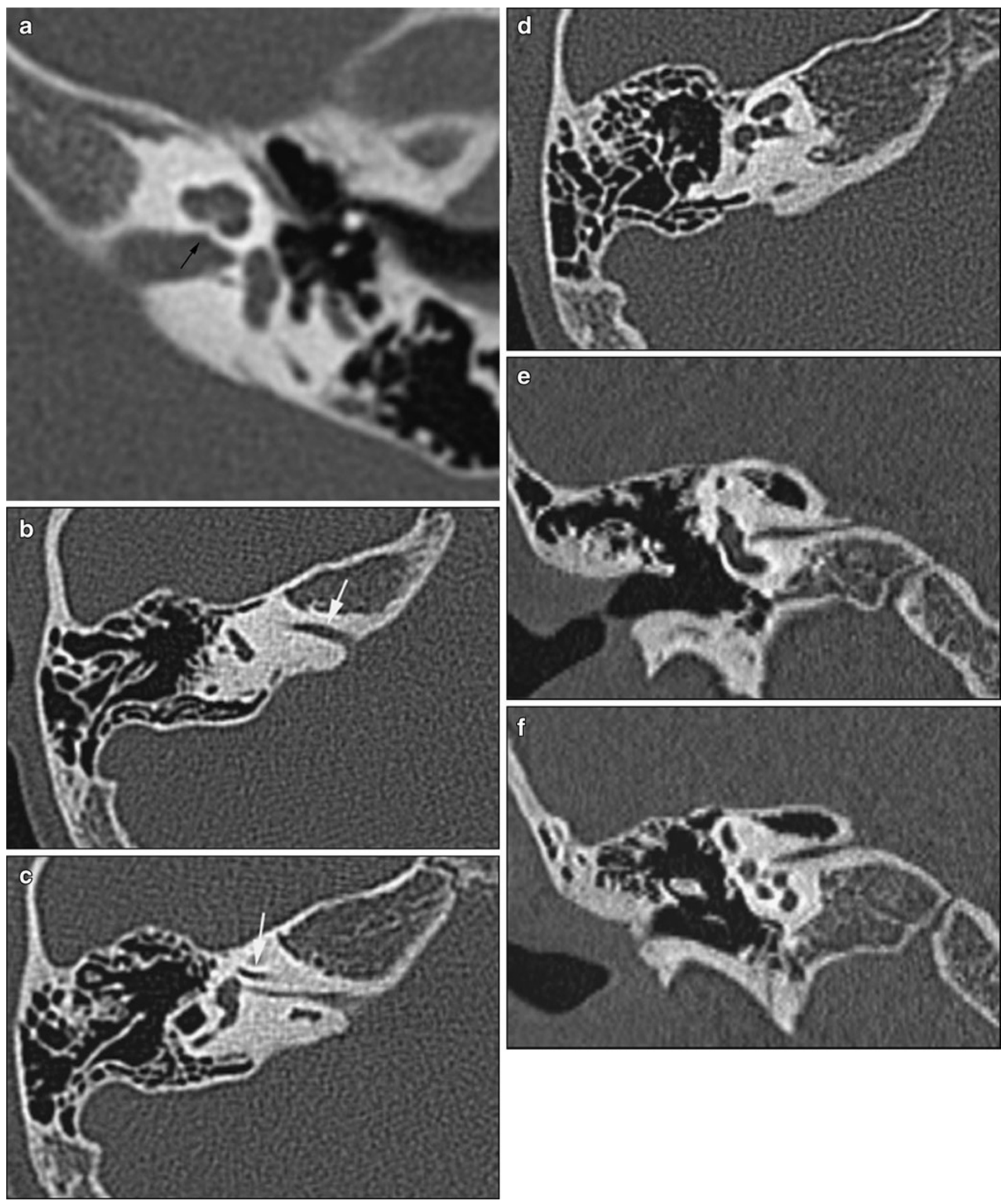

Fig. 9 Abnormal development of the internal auditory canal. a This child with congenital sensorineural hearing loss has a stenosis of the cochlear aperture (black arrow) on CT, indicative of a congenital malformation of the cochlear nerve. b-f Patient 2 shows a duplication

of the internal auditory canal. The separate canal for the facial nerve is indicated by a white arrow. The canal for the cochlear nerve is very narrow; the cochlear nerve is usually aplastic or hypoplastic in these cases 
On CT, obliteration of the cochlea will be accompanied by otospongiotic foci in the surrounding bone (Fig. 6). These may be difficult to visualise on MRI.

Intracochlear hemorrhage Intracochlear haemorrhage will cause increased signal intensity on native T1-weighted images. This is seen in the acute or subacute setting of trauma.

Intracochlear schwannoma Purely intralabyrintine schwannomas are rare. They are thought to arise from distal branches of the cochlear or vestibular nerves and are located in the cochlea, vestibule, semicircular canals or a combination of these locations. They have been reported to originate most often in the scala tympani. Growth into the scala vestibuli may occur later [11]. These patients present with tinnitus, vertigo and progressive hearing loss leading to a dead ear over time.

The mass causes focal signal loss on T2-weighted images, is slighty hyperintens to cerebrospinal fluid (CSF) on native T1-weighted images and shows strong enhancement on contrast-enhanced T1 images (Fig. 7). Both the clinical history and the persistent, focal, intense enhancement will help to distinguish from the more diffuse or multifocal enhancement in acute labyrinthitis.

Extension of a meatal, vestibular schwannoma into the inner ear is more frequently seen and will be discussed in the next section. If present in a patient considered for surgical removal of the tumour, this requires a transotic approach for complete removal [12].

\section{Internal auditory canal/cerebellopontine angle}

The imaging modality of choice to assess the internal auditory canal (IAC) and its content as well as the cerebellopontine angle (CPA) cistern is MRI. There is some controversy whether the imaging protocol should include contrast enhanced images [13, 14]. Although the yield of contrast-enhanced images will be low, it may reveal inflammatory, auto-immune or tumoural pathology of the inner ear, cochleovestibular nerve or meninges that might not be suspected clinically and impossible or difficult to see on native images. Therefore, we favour the use of contrast in the diagnostic work-up of hearing loss.

A typical imaging protocol is performed with a head coil and includes axial thin-section 3D T2-weighted images, T1-weighted images without and with contrast enhancement and coronal T1-weighted enhanced scan of the temporal bone as well as a whole-brain screen [fluid-attenuated inversion-recovery (FLAIR) or T2weighted]. A maximum section width of $3 \mathrm{~mm}$ with a minimal or no inter-slice gap and a small field of view (FOV) are needed [15].

Anatomy

The CPA cistern is an anatomic CSF-filled space located between the cerebellar peduncle, pons and
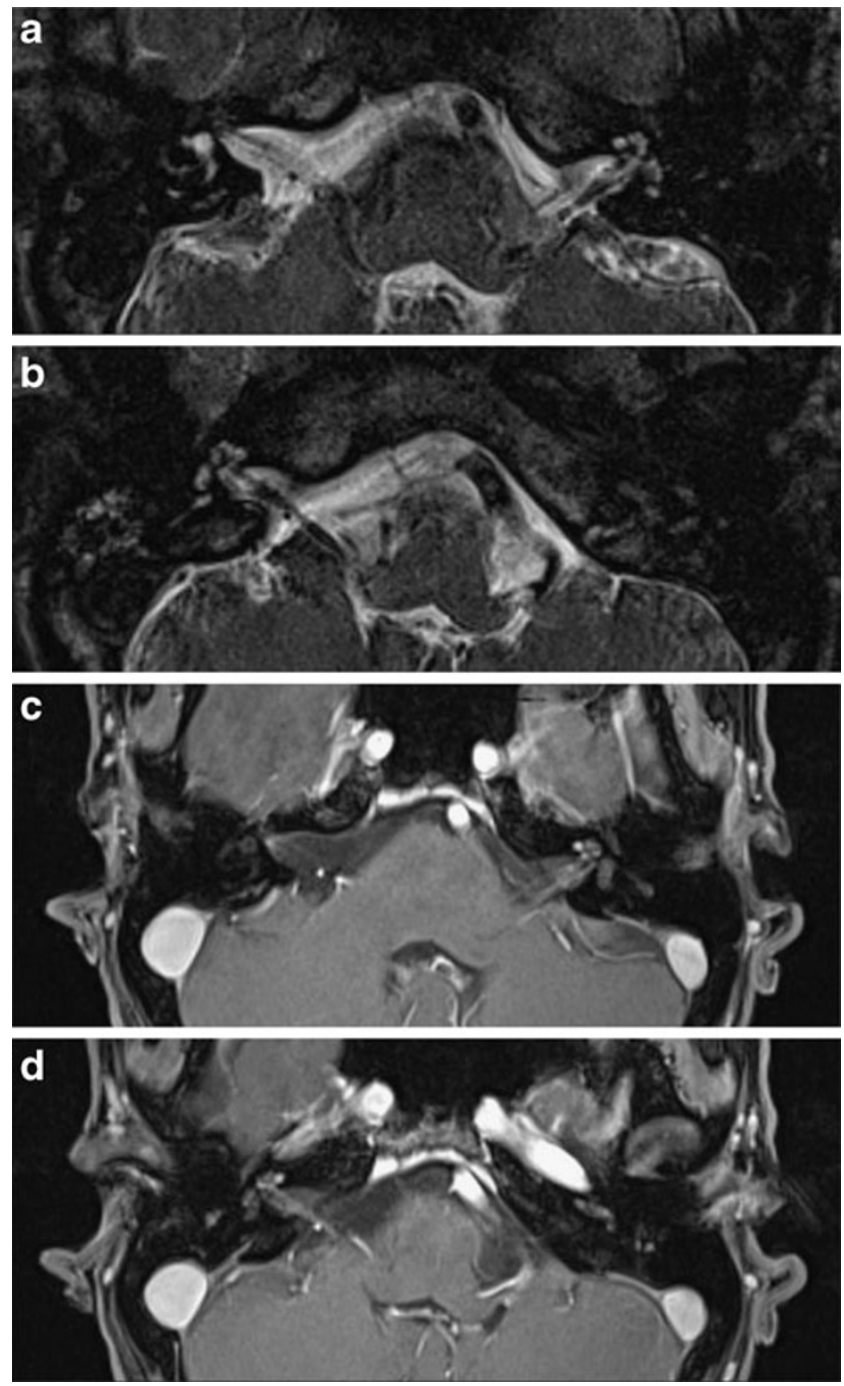

Fig. 10 Abnormal enhancement in the IAC due to leptomeningeal disease. This male adult presented with bilateral sensorineural hearing loss and asymmetric facial palsy. On MRI, thickening (a, b: 3D T2 TSE) and enhancement (c, d: contrast-enhanced T1) of the seventh and eighth cranial nerves as well as the cochlea is seen bilaterally, suggestive of a systemic infectious or inflammatory disease. Laboratory findings confirmed infection with Treponema pallidum. Labyrinthine involvement may occur in the secondary or primary stage of acquired syphilis, resulting from meningovascular and acute meningitic forms of neurosyphilis. Luetic syphilis may be accompanied by osteitis of the temporal bone. The permeative demineralisation of the bone may mimic the imaging appearance of otosclerosis, osteogenesis imperfecta or Paget's disease 

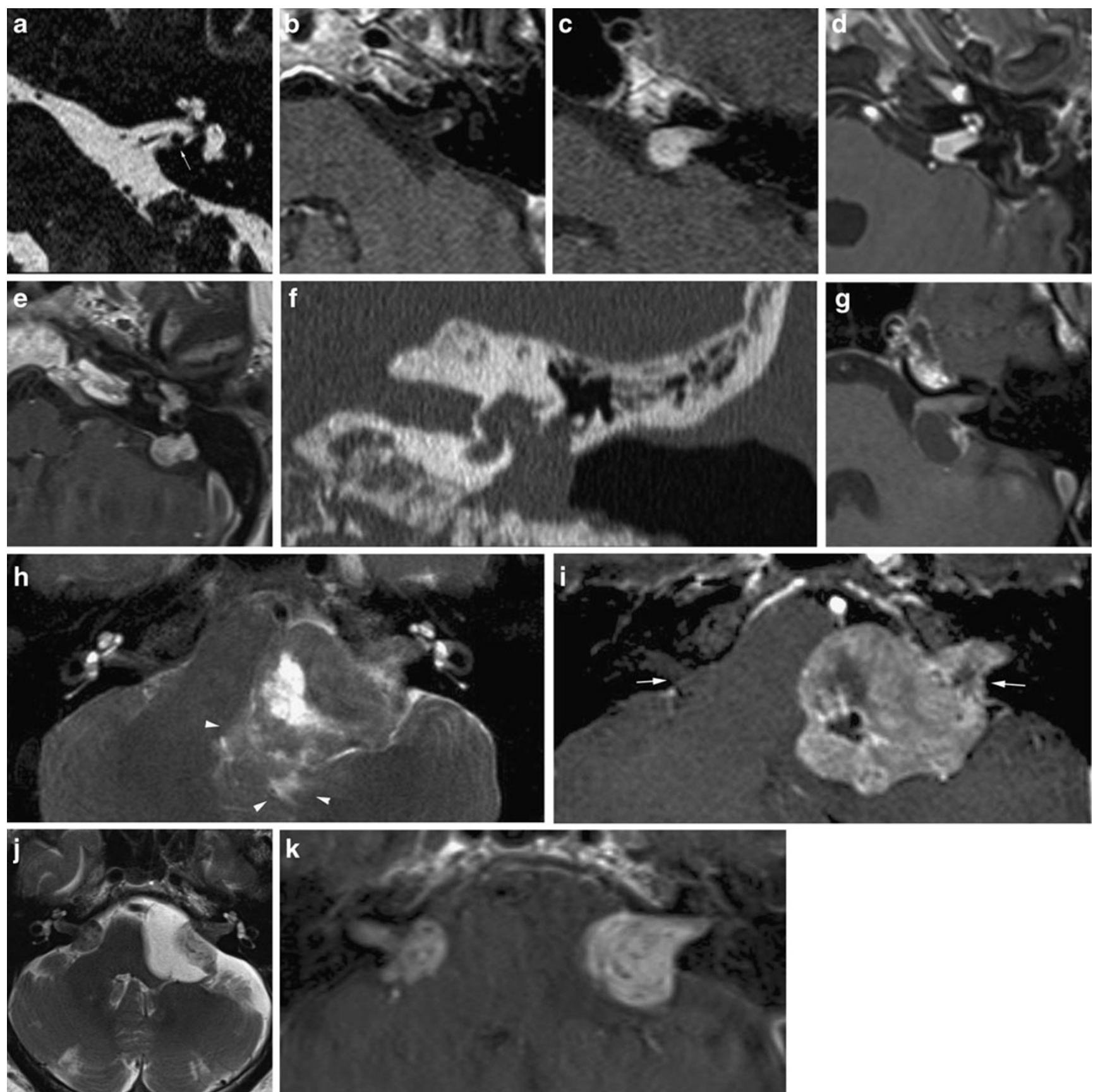

Fig. 11 Imaging features of vestibular schwannomas. A nodular lesion is seen along the inferior vestibular nerve (a) with strong enhancement (b). This is the typical site of origin of a schwannoma. c The typical growth pattern of a schwannoma is towards and extending into the CPA resembling an "ice-cream cone". d Less frequently, involvement of the inner ear is seen: in this case transmodiolar extension causes enhancement of the cochlea. e-f In rare cases extensive transotic growth may occur. In this patient, tumour involvement of the inner ear, endolymphatic duct and sac and the middle ear is present. Coronal CT (f) shows widening of the oval window, caused by the tumour extension to the middle ear cleft. $\mathbf{g}$ The extracanalicular portion of a schwannoma may show cystic degener- ation. In this patient the cyst formation is located posterior to the IAC, resulting in a somewhat atypical appearance eccentric to the porus. Such cyst formation seems to be associated with rapid growth. $\mathbf{h}, \mathbf{i}$ Further growth of a schwannoma will result in compression of the brain stem and cerebellum and eventually other cranial nerves such as the trigeminal nerve. In this patient compression of the fourth ventricle caused hydrocephalus (not shown). Note also the edema in the surrounding parenchyma (arrowheads on T2) and erosion of the porus (arrows on contrast enhanced T1). j, k Bilateral schwannomas are a hallmark of neurofibromatosis type 2. On the left, an arachnoid cyst has occured secondary to the mass lesion. Depending on the patient history, metastases should be considered as a differential diagnosis 
internal auditory canal. Several vessels and the facial and cochleovestibular nerve course through it. On its way to the internal auditory canal, the cochleovestibular nerve will divide into three branches: the cochlear nerve and the superior and inferior vestibular nerve supplying the cochlea and the vestibular system respectively. These branches lie posterior and inferior to the facial nerve (Fig. 8).

Imaging patterns of disease

\section{Abnormal development}

The formation of the IAC depends on the development of the cochleovestibular nerve. Mesoderm envelopes the eighth nerve and transforms into cartilage and bone to form the internal acoustic meatus [16]. Altered development of the eighth nerve will induce stenosis, atresia or duplication of the IAC [17-19] (Figs. 1d, e and 9). Abnormal development of the eighth nerve may also occur within a normal-sized IAC, either as aplasia or hypoplasia of the cochlear branch. The normal size of the cochlear nerve on MRI measures $1.8 \pm 0.2 \mathrm{~mm}$ at the porus acousticus and $1.2 \pm 0.2 \mathrm{~mm}$ in the mid to distal IAC; the vestibular nerves are smaller [20].

On CT, stenosis of the cochlear aperture at the fundus (Fig. 9) or narrowing of the IAC $(<2 \mathrm{~mm})$ indicates a cochlear nerve deficiency [21].

\section{Enhancement}

Enhancement along the course of the cochleovestibular nerve may be the result of viral infection (e.g. herpes simplex, herpes zoster, cytomegalovirus), bacterial infection spreading from the middle ear and mastoid or from a skull base osteomyelitis or may be seen as a manifestation of leptomeningeal disease (e.g. Lyme disease, sarcoidosis, syphillis, tuberculosis) (Fig. 10). Perineural spread of cancer presenting as isolated cochleovestibular enhancement is highly unlikely.

Infectious neuritis will cause enhancement of or around the eighth nerve and/or within the labyrinth. The edematous, inflamed nerve may present as a masslike lesion. In inflammatory neuritis, often multiple cranial nerves are affected. Associated findings of the underlying disease, such as brainstem involvement at the facial nerve root entry zone suggesting Lyme disease or meningeal enhancement as in tuberculous meningitis, should also be looked for.

\section{Mass lesion}

Within the IAC and CPA, soft tissue mass lesions or cystic mass lesions can be found.The soft tissue lesions

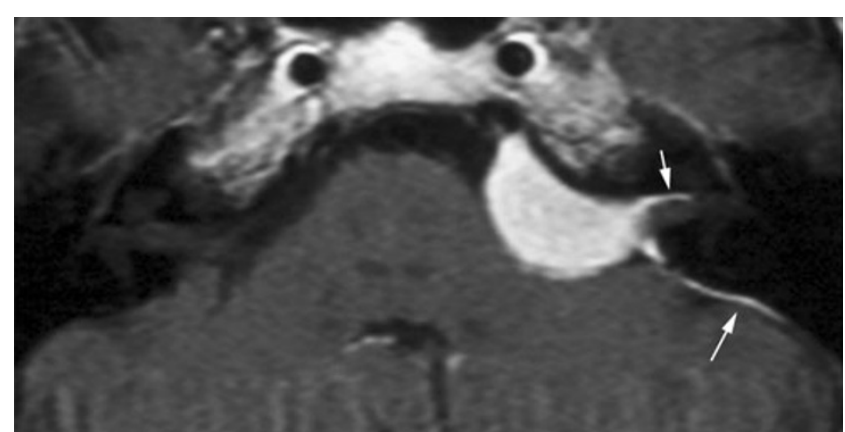

Fig. 12 Meningioma of the CPA. A mass lesion, eccentric to the porus and usually without extension into the internal acoustic meatus with obtuse angles towards a dural tail is the typical imaging appearance of a meningioma

are the most likely to cause vestibular symptoms or hearing loss. By far the most common soft tissue tumour in this area is the schwannoma (85\%). A meningioma occurs in about 3-5\%. Metastases, lipoma, vascular lesions or tumours from surrounding structures extending into the CPA are infrequent. The cystic lesions are congenital abnormalities: an epidermoid cyst results from epithelial rests and accumulation of fluid within the arachnoid membrane will lead to the formation of an arachnoid cyst.

\section{Soft tissue mass lesions}

Schwannoma Schwannomas arise from the inferior or superior vestibular nerve, near Scarpa's ganglion [22]. The imaging appearance is variable and depends on the growth pattern, size and histological features of the tumour (Fig. 11). The classic growth pattern shows growth along the eighth nerve, involving both the IAC and CPA. Less frequently the

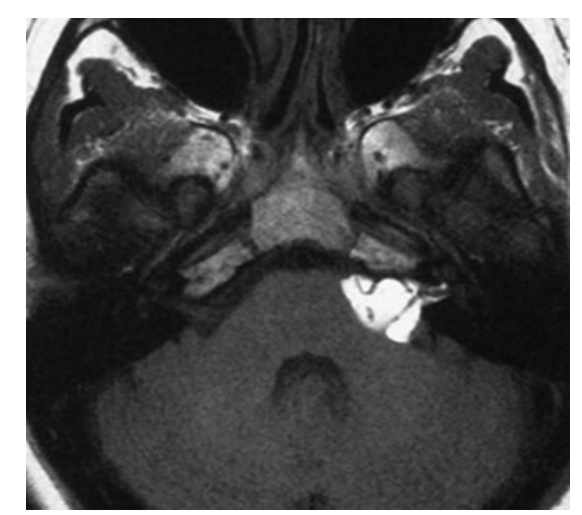

Fig. 13 Lipoma of the CPA. A hyperintens lesion on a non-contrastenhanced T1-weighted image with engulfment of neurovascular structures is consistent with a lipoma. (Courtesy of Dr. M. Lemmerling, AZ St Lucas, Gent, Belgium) 
tumour is confined to the CPA or IAC. The latter may extend into the labyrinth through the macula or modiolus [23]. With continued growth, mainly of the extracanalicular component, histology changes to a predominance of Antoni type B cells and cystic regression and necrosis will cause a cystic appearance of the tumour. Microhaemorrhages are a well-known pathological feature of schwannomas. These may be demonstrated on a $\mathrm{T} 2 *$-weighted gradient echo sequence[24]. There have been sporadic reports of spontaneous gross intratumoural bleeding in a vestibular schwannoma or leading to subarachnoidal haemorrhage [25-27]. These patients most often present with acute cranial neuropathies and symptoms of subarachnoid haemorrhage [25]. The likelihood for haemorrhage may be associated with preoperative radiotherapy and vascular abnormalities [26].

Schwannomas are typically centred on the porus and have a sharp angle towards the dura. Such an enhancing mass in the cone-shaped IAC, bulging into the CPA, resembles the image of an ice cream on a cone. Erosion of the porus may occur. Bilateral schwannomas should trigger the diagnosis of neurofibromatosis type II.

Menigioma Meningiomas arise from the dura along the petrous bone or the tentorium; hence these tumours are usually located eccentric to the porus with no or only limited involvement of the IAC. They usually have obtuse angles towards the dura, which is often thickened (dural tail) (Fig. 12). Calcifications are seen in up to $25 \%$.

Lipoma Lipomas of the IAC and CPA are rare. They present as a mass lesion with fatty signal on all sequences with engulfment of neural and vascular structures (Fig. 13).

Vascular lesions Vascular malformations causing hearing loss are rare. Hemangiomas, arising from the vascular plexus at Scarpa's ganglion, usually present with facial nerve palsy [28]. If small, tumours may mimic the far more
Fig. 14 Vascular lesions. A patient with progressive, pulsatile tinnitus, some hearing loss and a subtle facial nerve deficit was referred for imaging. MRI revealed a large, vividly enhancing mass with intralesional flow voids (a: axial $\mathrm{T} 1$; b: coronal post-contrast T1) at the fundus of the IAC, extending into the temporal fossa and middle ear. On CT, destruction of the surrounding bone is seen (arrows in c). Biopsy showed the lesion to be a hemangiopericytoma. This tumour is rarely seen intracranial and arises from pericytes, surrounding capillaries, in the meninges [29]. Only a few cases have been reported in the temporal bone. Patient 2 shows the typical imaging appearance of an arteriovascular malformation: flow voids due to enlarged feeding and draining vessels are present in the pons, CPA and prepontine cistern on T2 (d) with enhancement on post-contrast $\mathrm{T} 1$ (e)
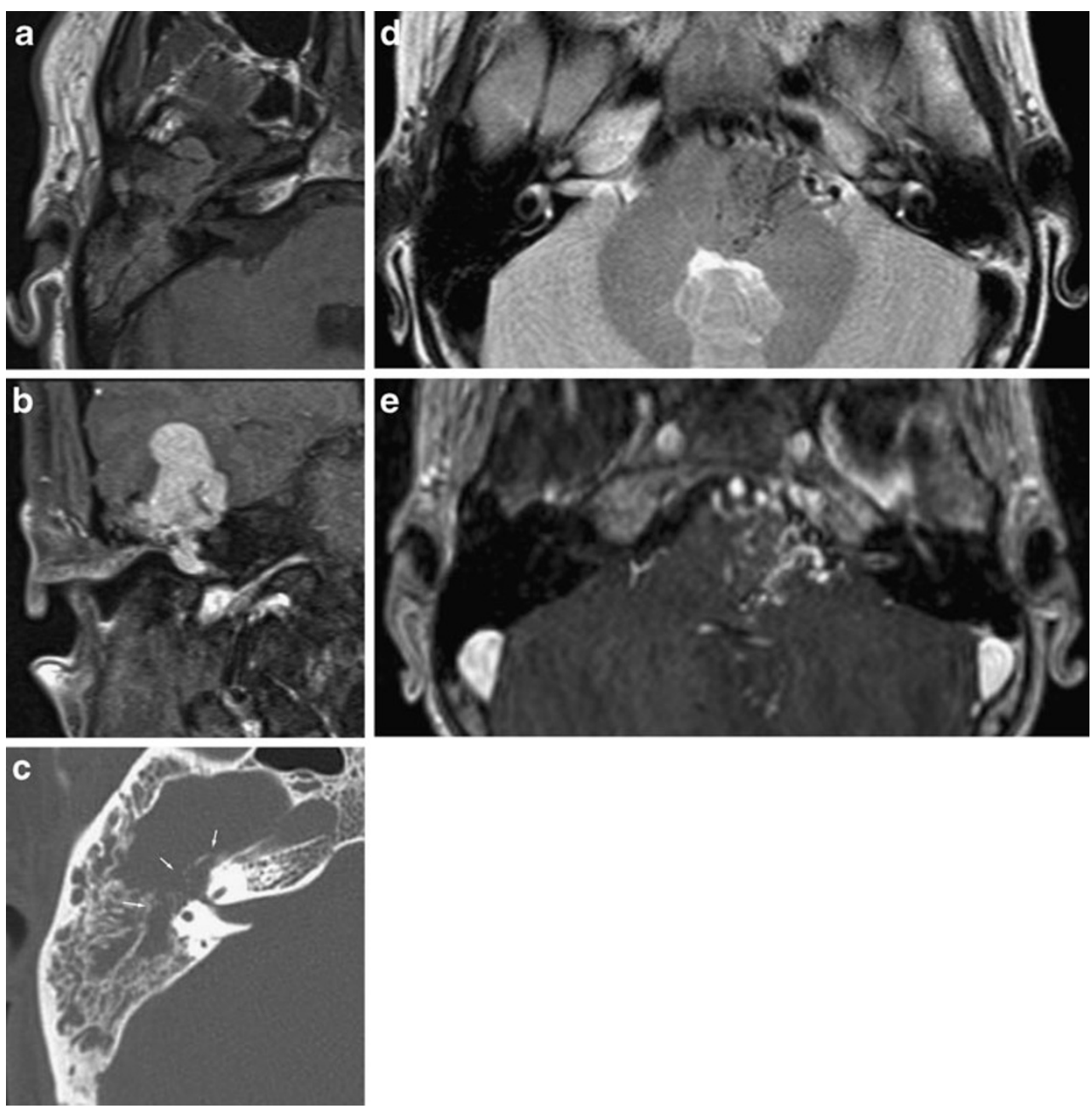
common schwannoma. There is, however, usually a descrepancy between tumour size and nerve VII and VIII deficit. Large vascular tumours have a tendency to erode surrounding bone. High-flow vascular malformations are usually accompanied with tinnitus and show fairly characteristic imaging findings (Fig. 14).

Metastases or tumours extending into the CPA Leptomeningeal or perineural tumour spread and lesions originating in the skull base, cerebellum or fourth ventricle may mimic vestibular schwannoma (Fig. 15). In case of metastatic disease there usually is a known history of malignancy. The most frequently encoutered primary sites are lung, breast, melanoma or lymphoma. Patients present with rapidly progressive symptoms, often combined with palsy of other cranial nerves, despite the small size of the lesion. Metastatic disease will show rapid growth.

\section{Cystic lesion}

Epidermoid cyst An epidermoid is a congenital lesion resulting from inclusion of ectodermal epithelial elements. However, they only become symptomatic when large enough to cause pressure-related complaints or cranial nerve palsy or headache. This is usually around the $3 \mathrm{rd}$ decade. The lesions may also be seen as an incidental finding in asymptomatic patients. Epidermoid cysts are well-defined lesions with a lobular or irregular nodular surface. They mimic a cystic lesion on T1- and T2weighted images, although the cyst is often hyperintense
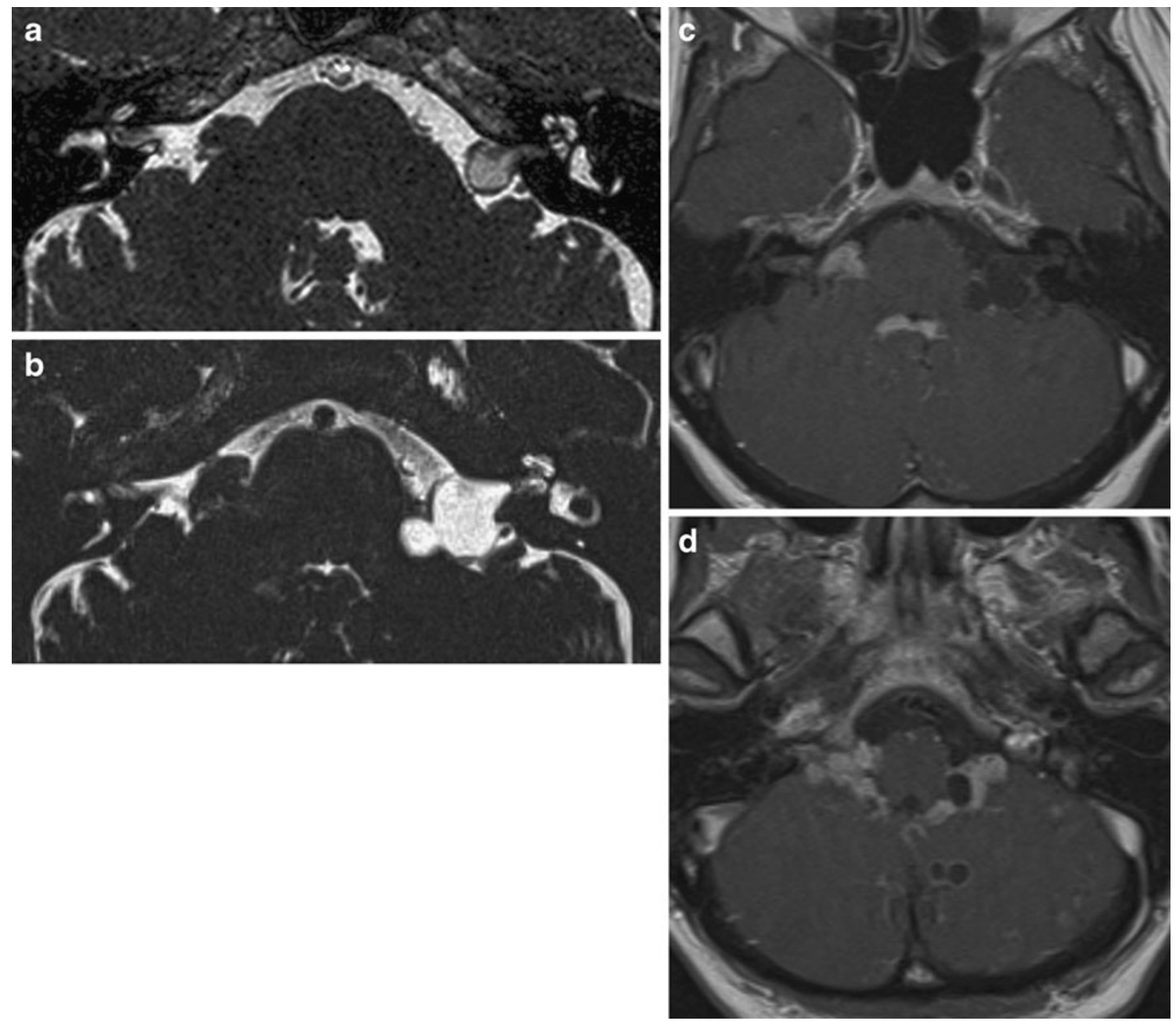

Fig. 15 Metastatic disease. A patient presenting with rapidly progressive hearing loss underwent MRI. On a heavily T2-weighted image (a) a tumour with an "ice-cream cone" appearance and cystic changes is present on the left. On the right a soft tissue mass is present in the cerebellopontine angle cistern. Follow-up examinta- tion a few months later (b-d) revealed extensive growth of the lesions, which are now evidently closely related to the choroid plexus. Postcontrast images (c, d) show cystic and nodular leptomeningeal metastases in the fossa posterior. CSF analysis showed malignant cells (adenocarcinoma) 

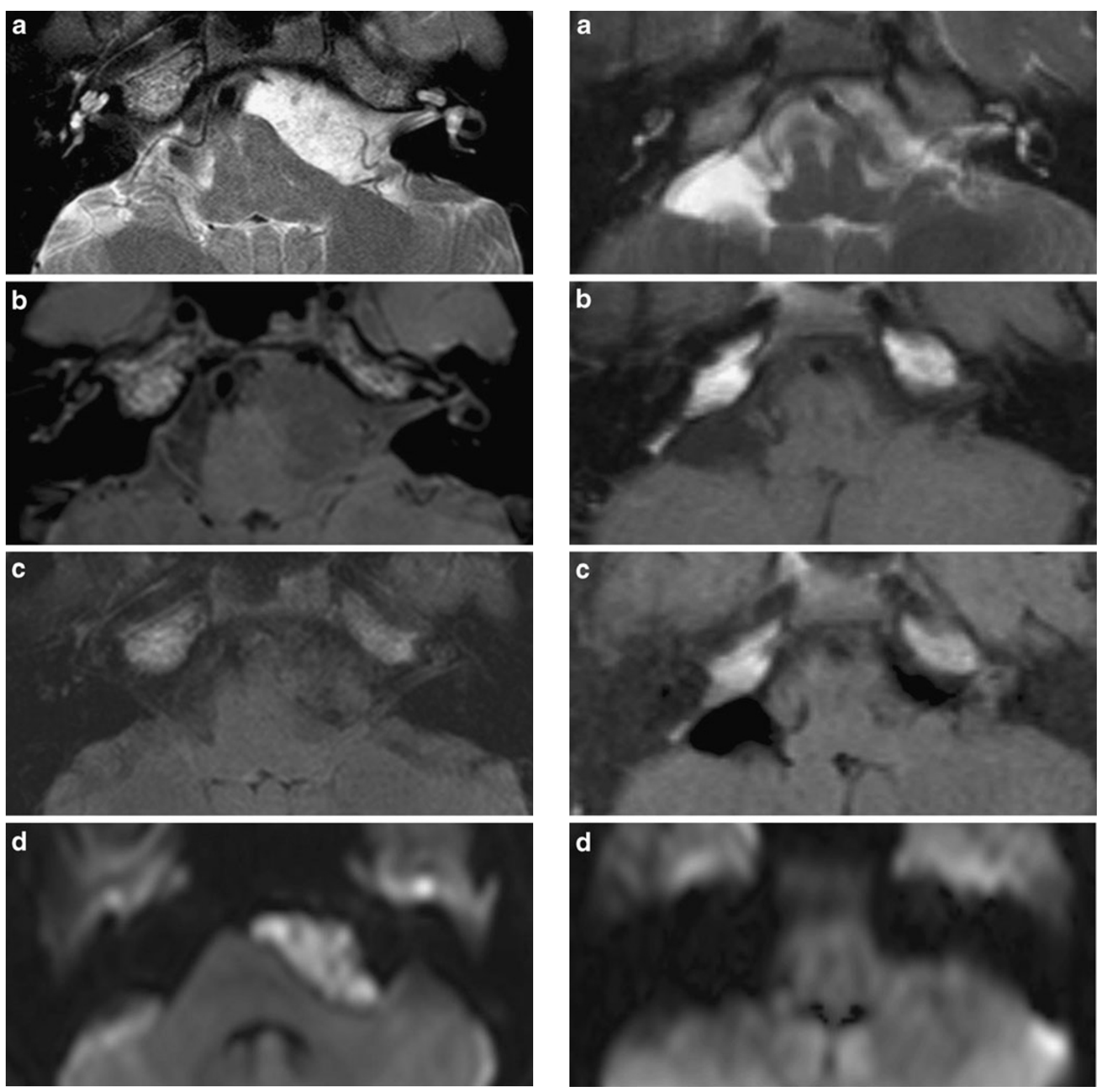

Fig. 16 Epidermoid cyst. On a T2-weighted image (a) a lesion is seen in the left cerebellopontine and prepontine cistern with a slithgly higher signal intensity than CSF. Note vessels running through the lesion. On T1 (b) a slightly increased signal intensity is seen, which is sometimes referred to as "dirty CSF". The incomplete supression of signal on FLAIR (c) and the diffusion restriction (d) confirm the

Fig. 17 Arachnoid cyst. On the right, a lesion with signal characteristics of fluid on all sequences (a: T2; b: T1; c: FLAIR; d: DWI) is present. Due to the relatively static fluid in the archanoid cyst the signal is higher than that of CSF on T2-weighted images diagnosis of an epidermoid cyst 
due to CSF on T2 and may show T1 shortening. FLAIR and diffusion-weighted imaging (DWI) sequences will show incomplete attenuation and diffusion restriction respectively (Fig. 16).

Arachnoid cyst The clinical presentation of arachnoid cysts resemble that of an epidermoid cyst. The distinguishing imaging feature is signal loss on FLAIR and the absence of diffusion restriction (Fig. 17). When large, arachnoid cysts may cause displacement of nerves and vessels and bone remodellation.

Other cystic lesions Other cystic lesions, such as a neurenteric or neuroepithelial cyst, are extremely rare.

\section{Conclusion}

Using apropriate imaging techniques and a pattern-based approach is helpful in detecting disease and narrowing the differential diagnosis in pathology of the inner ear and CPA cistern.

Open Access This article is distributed under the terms of the Creative Commons Attribution Noncommercial License which permits any noncommercial use, distribution, and reproduction in any medium, provided the original author(s) and source are credited.

\section{References}

1. American College of Radiology (2011) Practice guideline for CT of the head and neck: http://www.acr.org/SecondaryMain MenuCategories/quality_safety/guidelines/pediatric/ct_head neck.aspx, p 4

2. ten Donkelaar HJ, Lammens M, Hori A (2006) Clinical neuroembryology, 1st edn. Springer, Berlin Heidelberg New York, p 288

3. Bindu LH, Reddy PP (2008) Genetics of aminoglycoside-induced and prelingual non-syndromic mitochondrial hearing impairment: a review. Int J Audiol 47:702-707

4. Suehiro S, Sando I (1979) Congenital anomalies of the inner ear: introducing a new classification of labyrinthine anomalies. Ann Otol Rhinol Laryngol 88:1-24

5. Jackler RK, Luxford WM, House WF (1987) Congenital malformations of the inner ear: a classification based on embryogenesis. Laryngoscope 97:2-14

6. Sennaroglu L, Saatci I (2002) A new classification for cochleovestibular malformations. Laryngoscope 112:2230-2241

7. Zheng Y, Schachern PA, Cureoglu S, Mutlu C, Dijalilian H, Paparella MM (2002) The shortened cochlea: its classicification and histopathologic features. Int J Pediat Otorhinolaryngol 63:29 39
8. Merkus P, Free RH, Mylanus EA, Stokroos P, Metselaar M, Van Spronsen E, Grolman W, Frijns JH (2010) Dutch Cochlear Implant groups (CI-ON) consensus protocol on postmeningitis hearing evaluation and treatment. Otol Neurotol 31:1281-1286

9. Aschendorff A, Klenzner T, Laszig R (2005) Deafness after bacterial meningitis. An emergency for early imaging and cochlear implant surgery. Otolaryngol Head Neck Surg 133:995-996

10. Fayad J, Moloy P, Linthicum FH (1990) Cochlear otosclerosis: does bone formation affect cochlear implant surgery? Am J Otol 11:196-200

11. Tieleman A, Casselman JW, Somers T, Delanote J, Kuhweide R, Ghekiere J, De Foer B, Offeciers EF (2008) Imaging of intralabyrinthine schwannomas: a retrospective study of 52 cases with emphasis on lesion growth. AJNR Am J Neuroradiol 29:898-905

12. Falcioni M, Taibah A, Di Trapani G, Khrais T, Sanna M (2003) Inner ear extension of vestibular schwannomas. Laryngoscope 113:1605-1608

13. Jackler RK (1996) Cost-effective screening for acoustic neuroma with unenhanced MR: a clinician's perspective. AJNR Am J Neuroradiol 17:1226-1228

14. Annesley-Williams DJ, Laitt D, Jenkins JP, Ramsden RT, Gillespie JE (2001) MRI in the investigation of sensorineural hearing loss: is contrast enhancement still necessary? J Laryngol Otol 115:14-21

15. American College of Radiology (2007) Practice guideline for MRI of the head and neck: http://www.acr.org/SecondaryMainMenu Categories/quality_safety/guidelines/dx/head-neck/mri_head_ neck.aspx, p 5

16. Yates JA, Patel PC, Millman B, Gibson WS (1997) Isolated congenital internal auditory canal atresia with normal facial nerve function. Int J Pediatr Otorhinolaryngol 41:1-8

17. Giesemann AM, Neuburger J, Lanfermann H, Goetz F (2011) Aberrant course of the intracranial facial nerve in cases of atresia of the internal auditory canal (IAC). Neuroradiology 53:681-687

18. Ferreira T, Shayestehfar B, Lufkin R (2003) Narrow, duplicated internal auditory canal. Neuroradiology 45:308-310

19. Casselman JW, Offeciers FE, Govaerts PJ, Kuhweide R, Geldof H, Somers T, D'Hont G (1997) Aplasia and hypoplasia of the vestibulocochlear nerve: diagnosis with MR imaging. Radiology 202:773-781

20. Nadol JB Jr, Xu WZ (1992) Diameter of the cochlear nerve in deaf humans: implications for cochlear implantation. Ann Otol Rhinol Laryngol 101:988-993

21. Fatterpekar GM, Mukherji SK, Alley J, Lin Y, Castillo M (2000) Hypoplasia of the bony canal for the cochlear nerve in patients with congenital sensorineural hearing loss: initial observations. Radiology 215:243-246

22. Xenellis JE, L. Origin of vestibular schwannomas. Otol Neurotol 24:1

23. Salzman KL, Davidson HC, Harnsberger HR, Glastonbury CM, Wiggins RH, Ellul S, Shelton C (2001) Dumbbell schwannomas of the internal auditory canal. AJNR Am J Neuroradiol 22:13681376

24. Thamburai K, Radhakrishnan VV, Thomas B, Nair S, Menon G (2008) Intratumoral microhemorrhages on T $2 *$-weighted gradientecho imaging helps differentiate vestibular schwannoma from meningioma. AJNR Am J Neuroradiol 29:552-557

25. Carlson ML, Driscoll CL, Links MJ, Inwards CY, Neff BA, Beatty CW (2010) A hemorrhagic vestibular schwannoma presenting with rapid neurologic decline a case report. Laryngoscope 120:S204 
26. Dehdashti AR, Kiehl TR, Guha A (2009) Vestibular schwannomas presenting with haemorrhage: clinical presentations and histophological evaluation of an unusual entity. Br J Neurosurg 23:431-436

27. Gavra M, Thanos L, Pomoni M, Batakis N (2010) Spontaneous subarachnoid haemorrhage due to acoustic neurinoma: Case report and review of the literature. Br J Neurosurg 24:82-83
28. Avellino AM, Grant GA, Harris AB, Wallace SK, Shaw CM (1999) Recurrent intracranial Masson's vegetant intravascular hemangioendothelioma. Case report and review of the literature. J Neurosurg 91:308-312

29. Chin LS, Rabb CH, Hinton DR, Apuzzo ML (1993) Hemangiopericytoma of the temporal bone presenting as a retroauricular mass. Neurosurgery 33:728-731 